\title{
Bone Metastases in Breast Cancer
}

a report by

Allan Lipton, MD

Milton S Hershey Medical Center, Pennsylvania State University

DOI: $10.17925 /$ OHR.2006.00.00.1g

Patients with advanced breast cancer are at high risk for developing bone metastases and ensuing skeletal complications. Since the mid 1990s, intravenous (IV) pamidronate has been the international standard of care for breast cancer patients with bone metastases. Recently, a large, randomized, phase III trial in this patient population demonstrated that $4 \mathrm{mg}$ zoledronic acid, a new-generation bisphosphonate, was safe and significantly reduced the overall risk of skeletal complications compared with pamidronate. Zoledronic acid has also been shown to provide consistent, durable pain reduction in these patients compared with placebo. Based on these results, zoledronic acid is rapidly becoming the new international standard for patients with bone metastases from breast cancer.

Introduction

More than 200,000 women in the US are diagnosed with breast cancer each year ${ }^{1}$ and, among the approximate one-third of these patients who develop advanced disease, $65-75 \%$ will develop bone metastases. ${ }^{2}$ These patients are at high risk for skeletal complications, including pathologic fractures, spinal cord compression, and debilitating bone pain. In fact, studies have demonstrated that in the absence of bisphosphonate therapy nearly $70 \%$ of breast cancer patients with bone metastases will experience a skeletal complication within two years, with an annual incidence of approximately four events per year (see Figure 1) ${ }^{3,4}$ Moreover, breast cancer patients have a long median survival after diagnosis of bone metastases (in the range of two to three years), during which they often suffer from significant skeletal morbidity that can have a devastating effect on quality of life. . $^{2,5}$

Traditional options for the treatment of patients with bone metastases from advanced breast cancer include standard anti-neoplastic therapies, surgery to treat or prevent fractures, and radiotherapy to stabilize bone lesions, prevent fractures, and palliate severe bone pain. In addition, these patients often receive systemic analgesic therapy with non-steroidal anti-inflammatory drugs (NSAIDS) or opioids. Recently, bisphosphonates have emerged as the standard for the prevention of skeletal complications in breast cancer patients with bone metastases. Bisphosphonates are potent inhibitors of bone resorption and have been shown to significantly reduce the percentage of patients with skeletal complications, delay the median time to first skeletal event, reduce the need for radiotherapy, and palliate bone pain compared with placebo in randomized, controlled trials. ${ }^{6}$ Currently, IV pamidronate $(90 \mathrm{mg})$ and zoledronic acid $(4 \mathrm{mg})$ are the only bisphosphonates recommended by the American Society of Clinical Oncology (ASCO) for the treatment of patients with multiple myeloma or breast cancer, ${ }^{7,8}$ and zoledronic acid is quickly becoming the new standard of care for the treatment of bone metastases in patients with breast cancer.

The Rolle of Bisphosphonates in Breast Cancer

Both IV and oral bisphosphonates have been shown to provide significant clinical benefits in patients with bone metastases from breast cancer in randomized trials (see Table 1). ${ }^{3,9-16}$ Conservative assessments of treatment effects include first-event analyses, such as percentage of patients with at least one skeletal complication or time to first skeletal complication, and are preferred end-points for regulatory approval by the US Food and Drug Administration (FDA) ${ }^{17}$ Treatment effect on incidence of pathologic fractures is particularly important. Oral bisphosphonates have not demonstrated consistent, statistically significant reductions in the percentage of patients with at least one skeletal complication. In addition, the use of oral bisphosphonates is complicated by poor bioavailability and gastrointestinal (GI) toxicity, as well as issues with patient compliance. ${ }^{18}$ Currently, these agents are only approved outside the US for the treatment of bone metastases from breast cancer. ${ }^{19}$ This section provides a brief review of the efficacy and safety of IV and oral bisphosphonates currently marketed for the treatment of patients with bone metastases from breast cancer.

\section{Clodronate}

Studies investigating the efficacy of oral clodronate $(1,600 \mathrm{mg} /$ day $)$ in patients with bone metastases from breast cancer have demonstrated significant clinical benefits compared with placebo or controls. ${ }^{12,13}$ However, 
Figure 1: Percentage of Patients with Each Type of SRE in the Placebo Group of Two Pooled Pamidronate Trials after Two Years of Follow-up

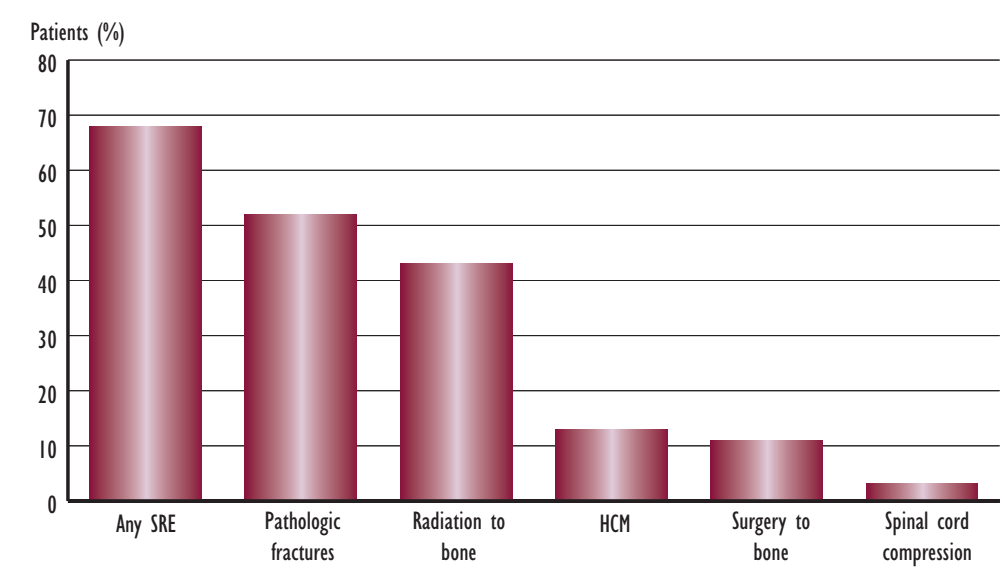

HCM = Hypercalcemia of malignancy. Data from Lipton et al. ${ }^{3}$ Adapted with permission from Coleman. ${ }^{4}$

these studies are several years old, and patient populations and chemotherapeutic regimens have since changed, making comparisons to newer bisphosphonates difficult. In a study involving 173 patients, treatment with clodronate for 18 months significantly reduced the incidence of all skeletal events, as well as the incidence of vertebral fractures and the rate of vertebral deformity, compared with placebo $(\mathrm{p}<0.025) .{ }^{12}$ However, the efficacy of oral clodronate was complicated by a lack of compliance during this trial. Of the $78 \%$ of complianceevaluable patients, $26 \%$ were completely non-compliant with the oral regimen. Similarly, in a randomized, openlabel trial involving 100 patients being treated with chemotherapy or endocrine therapy, treatment with concomitant clodronate for two years significantly prolonged the time to first skeletal event $(p=0.015)$ and reduced the incidence of fractures $(p=0.023)$ compared with patients who did not receive clodronate. ${ }^{13}$ However, the efficacy of clodronate was transient; after 15 months, the need for radiotherapy to treat bone pain increased in the clodronate group compared with the control group. Recent studies have also investigated the efficacy of oral clodronate for the prevention of bone metastases in patients with breast cancer. Although the study reported by Powles et al..$^{20}$ demonstrated positive results, this outcome has not been confirmed by other trials that did not show any clinical benefit of oral clodronate for this indication. ${ }^{21,22}$

Pamidronate

Since the mid 1990s, IV pamidronate (90mg via twohour infusion) has been the standard of care for breast cancer patients with bone metastases. Pamidronate became the gold standard on the basis of evidence from two long-term, randomized, placebo-controlled trials that demonstrated that this agent significantly reduced the incidence and delayed the onset of skeletal-related events (SREs). ${ }^{3,23,24}$ These studies defined an SRE as a pathologic fracture, spinal cord compression, or radiation or surgery to bone. Hypercalcemia was also included as an SRE for some assessments. In these trials, bone pain was also measured quantitatively using a scoring system that assessed both the severity and frequency of bone pain.

A pooled analysis of these two trials demonstrated that treatment with pamidronate resulted in statistically significant reductions in all types of skeletal complications and bone pain compared with placebo. ${ }^{3}$ In these trials, 751 patients were treated with pamidronate (90mg) or placebo every three to four weeks. After 24 months of therapy, pamidronate significantly reduced the percentage of patients who experienced at least one SRE excluding hypercalcemia (51\% versus $64 \%$ for placebo; $\mathrm{p}<0.001$; see Figure 2) $)^{3,6,9}$ and the annual incidence of SREs (2.4 versus 3.7 SREs/year for placebo; $p<0.001$ ), and significantly delayed the median time to the first SRE by more than five months (12.7 versus seven months for placebo; $\mathrm{p}<0.001)$. At 24 months, pamidronate also significantly reduced mean pain scores $(p=0.015$ versus placebo) and analgesic scores $(p<0.001$ versus placebo) from baseline compared with increases from baseline in the placebo group. Moreover, these trials validated the composite SRE end-point and set a new standard for clinical evaluation of bisphosphonates for the treatment of malignant bone disease.

\section{Ibandronate}

Recently, IV ibandronate was approved in Europe for the treatment of bone metastases in patients with breast cancer, on the basis of a randomized trial comparing IV ibandronate (2mg or $6 \mathrm{mg}$ ) with placebo. ${ }^{11}$ This study defined bone events as vertebral fractures, pathologic non-vertebral fractures, and radiation or surgery to bone. In this trial involving 466 patients, IV ibandronate $(6 \mathrm{mg}$ via one- to two-hour infusion) every three to four weeks for up to 96 weeks significantly reduced the skeletal morbidity period rate (SMPR) — defined as the number of 12 -week periods with a new bone event divided by number of periods on study-compared with placebo $(p=0.004)$ and significantly delayed the median time to first bone event (50.6 versus 33.1 weeks for placebo; $\mathrm{p}=0.018) .{ }^{11}$ In contrast, the $2 \mathrm{mg}$ bolus IV dose was not effective. In addition, at last evaluation, IV ibandronate (6mg) significantly reduced mean pain scores (assessed on a 5-point scale) from baseline compared with increased pain scores in both the $2 \mathrm{mg}$ ibandronate and placebo groups $\left(\mathrm{p}<0.001\right.$ versus placebo). ${ }^{25}$

Recently, studies have also demonstrated the clinical benefit of oral ibandronate in preventing skeletal complications in breast cancer patients with bone metastases. Pooled results from two randomized studies involving 564 patients demonstrated that oral ibandronate $(50 \mathrm{mg} /$ day for 96 weeks) significantly reduced the SMPR compared with placebo $(\mathrm{p}=0.004) .{ }^{14}$ 
Table I: Clinical Trial Results for Bisphosphonates in Patients with Bone Metastases Secondary to Breast Cancer

Triallend-point

Key clinical comparison

$P$ value

\begin{tabular}{|c|c|c|c|}
\hline \multirow{2}{*}{\multicolumn{4}{|c|}{ IV bisphosphonates }} \\
\hline & & & \\
\hline \multirow[t]{2}{*}{ Rosen et al., $2003^{9}$} & Zoledronic acid $4 m g$ & Pamidronate $90 \mathrm{mg}$ & \\
\hline & $(n=378) *$ & $(n=388)^{*}$ & \\
\hline Patients with an SRE, \% & 46 & 49 & NS \\
\hline Median time to first SRE, days & $376 t$ & $366 t$ & NS \\
\hline Skeletal morbidity rate, SREs/year & 0.91 & 1.57 & 0.102 \\
\hline Multiple event analysis, hazard ratio & 0.799 & - & 0.025 \\
\hline \multirow[t]{2}{*}{ Kohno et al., $2004^{10}$} & Zoledronic acid $4 \mathrm{mg}$ & Placebo & \\
\hline & $(n=|| 4)$ & $(n=I \mid 3) \ddagger$ & \\
\hline Patients with an SRE, \% & 30.7 & 52.2 & 0.001 \\
\hline Median time to first SRE, days & Not reached & 360 & 0.004 \\
\hline SRE rate, events/patient-years & 0.63 & 1.10 & 0.016 \\
\hline Multiple event analysis, hazard ratio & 0.56 & - & 0.009 \\
\hline \multirow[t]{2}{*}{ Lipton et al., $2000^{3}$} & Pamidronate $90 \mathrm{mg}$ & Placebo & \\
\hline & $(n=367)$ & $(n=384)$ & \\
\hline Patients with an SRE, \% & 51 & 64 & $<0.001$ \\
\hline Median time to first SRE, months & 12.7 & 7.0 & $<0.001$ \\
\hline Skeletal morbidity rate, SREs/year & 2.4 & 3.7 & $<0.001$ \\
\hline \multirow[t]{2}{*}{ Body et al., 2003"I } & Ibandronate $6 \mathrm{mg}$ & Placebo & \\
\hline & $(n=154)$ & $(n=158)$ & \\
\hline Patients with an SRE, \% & 51 & 62 & NS \\
\hline Median time to first SRE, months & 11.8 & 7.7 & 0.018 \\
\hline SMPR, intervals/year & 1.19 & 1.48 & 0.004 \\
\hline \multicolumn{4}{|l|}{ Oral bisphosphonates } \\
\hline \multirow[t]{2}{*}{ Paterson et al., $1993^{12}$} & Clodronate $1,600 \mathrm{mg}$ & Placebo & \\
\hline & $(n=85)$ & $(n=88)$ & \\
\hline Mean SMR, events/I00 patient-years & 218.6 & 304.8 & $<0.001$ \\
\hline Vertebral fractures//00 patient-years & 84 & 124 & $<0.025$ \\
\hline Vertebral deformity/l00 patient-years & 168 & 252 & $<0.001$ \\
\hline \multirow[t]{2}{*}{ Kristensen et al., 1999/3 } & Clodronate $1,600 \mathrm{mg}$ & No drug & \\
\hline & $(n=49)$ & $(n=5 I)$ & \\
\hline Patients with a skeletal event, \% & 28.6 & 41.2 & NS \\
\hline Time to first skeletal event & Not reported & Not reported & 0.015 \\
\hline \multirow[t]{2}{*}{ Body et al., $2004^{14}$} & Ibandronate $50 \mathrm{mg}$ & Placebo & \\
\hline & $(n=287)$ & $(n=277)$ & \\
\hline Patients with new bone event, $\%$ & 45 & 52 & NS \\
\hline Median time to new bone event, months & 21.1 & 15.1 & NS \\
\hline Mean SMPR & 0.95 & 1.18 & 0.004 \\
\hline Poisson regression, hazard ratio & 0.62 & - & $<0.001$ \\
\hline
\end{tabular}

SRE=skeletal-related event; $N S=$ not significant; $S M P R=$ skeletal morbidity period rate.

*Intent-to-treat population based on Rosen et al. ${ }^{15}$

tData from Rosen et al. ${ }^{16}$

¥One patient did not receive study treatment and was excluded from efficacy and safety analyses.

However, it did not significantly reduce the percentage of patients with a new bone event, significantly delay the time to first bone event, or significantly reduce the number of skeletal fractures. Moreover, the percentage of patients reporting a GI adverse event (including dyspepsia, nausea, abdominal pain, and esophagitis) was higher in patients treated with oral ibandronate compared with placebo (14.6\% versus $7.6 \%$ for placebo). Additionally, pain assessments demonstrated that oral ibandronate was effective for palliation of bone pain throughout the course of the study. At last evaluation, ibandronate significantly reduced mean pain scores (assessed on a 5-point scale) from baseline compared with increased pain scores in patients receiving placebo $\left(p=0.001\right.$ versus placebo). ${ }^{26}$ Of note, although both IV and oral ibandronate have demonstrated some clinical benefit in breast cancer patients with bone metastases, neither agent has been directly compared with IV pamidronate or zoledronic acid. Furthermore, IV and oral ibandronate have never been directly compared with each other. In order to assess the clinical benefit of these agents, these comparisons are needed. ${ }^{27,28}$ 
Figure 2: Percentage of Patients with at Least One SRE (Excluding Hypercalcemia of Malignancy) in Breast Cancer Patients Treated with either Placebo Versus $90 \mathrm{mg}$ Pamidronate or $90 \mathrm{mg}$ Pamidronate Versus $4 \mathrm{mg}$ Zoledronic Acid

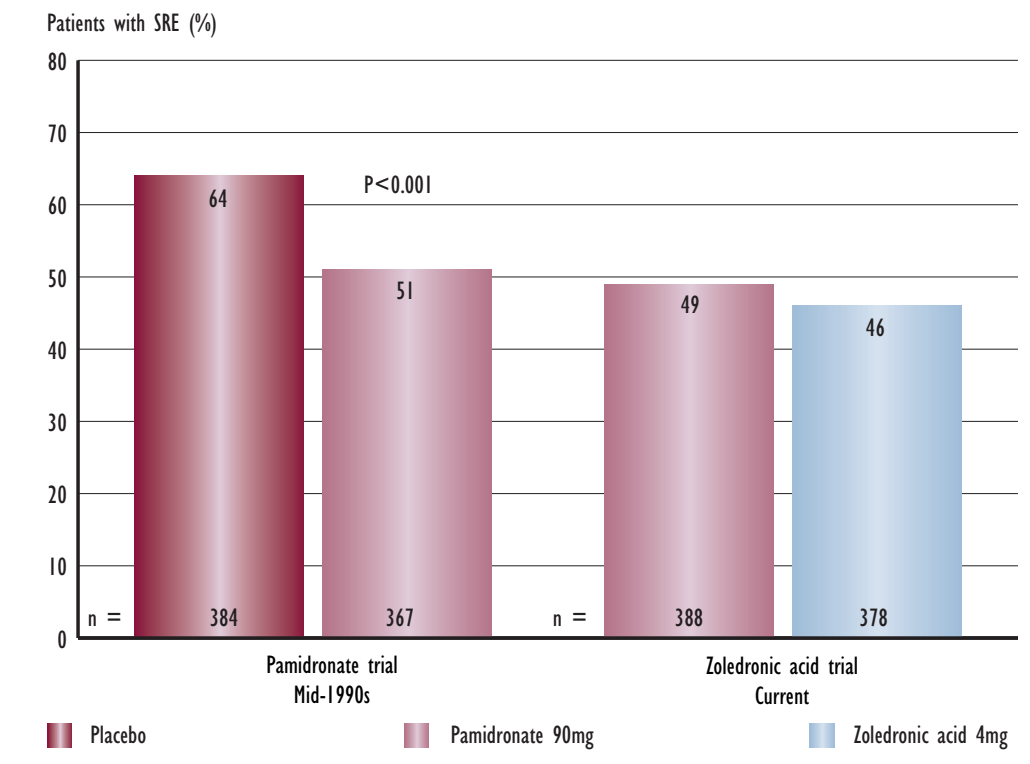

Data from Lipton et al. ${ }^{3}$ and Rosen et al. ${ }^{9}$ Adapted with permission from Coleman. ${ }^{6}$

Zoledronic Acid

The randomized phase III trial of zoledronic acid involved patients with bone lesions associated with breast cancer or multiple myeloma, and was a noninferiority trial directly comparing zoledronic acid with pamidronate, the standard of care at the time. Like the pamidronate trials previously discussed, the zoledronic acid trial assessed efficacy using the composite SRE end-point, and evaluation of the primary end-point (percentage of patients with at least one SRE) excluded hypercalcemia from the definition of SREs. In addition, this trial included a planned multiple event analysis using the Andersen-Gill method, ${ }^{29}$ which provides a comprehensive assessment of skeletal morbidity based on both the incidence and timing of all SREs. Bone pain was assessed by measuring mean change from baseline in brief pain inventory (BPI) scores.

Results from this study demonstrated that zoledronic acid (4mg) was at least as effective as pamidronate (90mg) in the overall patient population and was significantly more effective at reducing the risk of developing an SRE (by Andersen-Gill analysis) compared with pamidronate in the overall patient population. ${ }^{9,30}$ Moreover, zoledronic acid demonstrated superiority to pamidronate in the subset of patients with breast cancer. A total of 766 breast cancer patients with bone metastases were randomized to zoledronic acid (4mg via 15-minute infusion) or pamidronate (90mg via two-hour infusion) every three to four weeks for up to 24 months. At the final analysis at 25 months, the percentage of patients who developed at least one SRE was similar between treatment groups ( $46 \%$ for zoledronic acid versus $49 \%$ for pamidronate), which is consistent with the results of the placebocontrolled pamidronate trials previously discussed (see Figure 2)., ${ }^{3,69}$ However, zoledronic acid consistently reduced the percentage of patients with each type of SRE, ${ }^{16}$ and the Andersen-Gill multiple event analysis showed that treatment with zoledronic acid significantly reduced the overall risk of experiencing an SRE by an additional 20\% compared with pamidronate (hazard ratio $=0.799 ; \mathrm{p}=0.025) .{ }^{9}$ Both zoledronic acid and pamidronate also decreased pain scores in this trial. Among patients with pain scores $>0$ at baseline, mean composite BPI pain scores decreased from baseline at every time-point up to month 13 (the last time-point assessed) regardless of treatment. ${ }^{30}$ Moreover, at 25 months, significantly fewer patients treated with zoledronic acid required radiotherapy to bone, which can be considered a surrogate for bone pain, compared with pamidronate $(19 \%$ versus $27 \%$ for pamidronate; $\mathrm{p}=0.011) .{ }^{16}$ Importantly, zoledronic acid $(4 \mathrm{mg})$ was well tolerated; flu-like symptoms were more common in patients treated with zoledronic acid compared with placebo, but these were mild and occurred primarily after the first infusion.

Recently, a randomized, placebo-controlled study was conducted to investigate the efficacy of zoledronic acid in 228 Japanese women with bone metastases from breast cancer. ${ }^{10}$ In this trial, patients were treated with zoledronic acid ( $4 \mathrm{mg}$ every four weeks) or placebo for one year. The primary end-point in this trial was the ratio of the SRE rate (defined as the total number of SREs divided by time on study) for patients treated with zoledronic acid versus placebo. Similar to the other trial, this primary efficacy analysis did not include hypercalcemia in the definition of SREs. Results demonstrated that zoledronic acid significantly reduced the SRE rate by $43 \%$ compared with placebo $(p=0.016)$. Zoledronic acid also significantly reduced the percentage of patients who developed an SRE (31\% versus $52 \%$ for placebo; $p=0.001$ ), delayed the onset of skeletal complications $(p=0.004)$, and reduced the overall risk of developing an SRE by $44 \%$ (hazard ratio $=0.56 ; \mathrm{p}=0.009)$ compared with placebo. Moreover, zoledronic acid provided durable reductions of mean BPI pain scores from baseline and compared with the placebo group throughout the study. Patients treated with zoledronic acid experienced a statistically significant decrease from baseline in their mean pain score at every time-point beyond week two, including the 12-month time-point ( $p=0.0004)$.

Although all IV and oral bisphosphonates have been shown to reduce the incidence of skeletal complications compared with placebo, observation of a single endpoint used in the randomized, placebo-controlled trials 
discussed above in similar populations of breast cancer patients with bone metastases demonstrates that zoledronic acid significantly reduced the percentage of patients with at least one SRE by $40 \%$ relative to placebo at one year $(p=0.001) \cdot{ }^{10}$ IV pamidronate (90mg) significantly reduced, but to a lesser extent, the number of patients experiencing at least one SRE by approximately $22 \%$ relative to placebo at two years $(\mathrm{p}<0.001),{ }^{3}$ and both IV ibandronate $(6 \mathrm{mg})$ and oral ibandronate $(50 \mathrm{mg} /$ day) failed to significantly reduce the percentage of patients with a new bone event relative to placebo at one year $(18 \%$ and $13 \%$ relative reductions, respectively). ${ }^{11,14}$

\section{Conclusion}

Patients with advanced breast cancer are at high risk for developing bone metastases, which often result in painful skeletal complications that can negatively affect quality of life. Both oral and IV bisphosphonates provide clinical benefits for these patients; however, IV bisphosphonates have demonstrated more consistent and durable benefits without the GI toxicity, variable absorption, and lack of compliance associated with oral bisphosphonates. Current ASCO guidelines recommend the use of either IV pamidronate or zoledronic acid for patients with advanced breast cancer and bone metastases. ${ }^{7}$ However, zoledronic acid has demonstrated superior efficacy compared with pamidronate in patients with breast cancer, and zoledronic acid can be administered via a more convenient 15-minute infusion. Zoledronic acid has therefore rapidly become the new international standard of care for the treatment of bone metastases in patients with breast cancer. Currently, studies are ongoing to investigate the role of oral and IV bisphosphonates for the prevention of cancer treatment-induced bone $\operatorname{loss}^{31,32}$ and the prevention of bone metastases..$^{33}$ Recently, analysis of a large, five-year, multicenter trial demonstrated that oral clodronate significantly reduced the incidence of bone metastases in patients with primary operable stage I to III breast cancer compared with placebo. ${ }^{20}$ Therefore, the role of bisphosphonates in the treatment of breast cancer is likely to expand. In the future, bisphosphonates will not only be administered for the prevention of skeletal morbidity in patients with advanced metastatic disease, but may also be prescribed as early adjuvant therapy for the maintenance of bone health in these patients throughout their continuum of care.

References

1. Jemal A, Tiwari R C, Murray T et al., "Cancer statistics", CA Cancer J. Clin. (2004), 54: pp. 8-29.

2. Coleman R E, "Metastatic bone disease: clinical features, pathophysiology and treatment strategies", Cancer Treat Rev. (2001);27: pp. 165-176.

3. Lipton A, Theriault $R$ L, Hortobagyi G N et al., "Pamidronate prevents skeletal complications and is effective palliative treatment in women with breast carcinoma and osteolytic bone metastases: long term follow-up of two randomized, placebo-controlled trials", Cancer (2000);88: pp. 1,082-1,090

4. Coleman R E, "The role of bisphosphonates in breast cancer", The Breast (in press).

5. Weinfurt K P, Castel L D, LiY,Timbie JW, Glendenning GA, Schulman KA, "Health-related quality of life among patients with breast cancer receiving zoledronic acid or pamidronate disodium for metastatic bone lesions”, Med. Care (2004);42: pp. 164-175.

6. Coleman R E, "Bisphosphonates: clinical experience", Oncologist (2004);9 (suppl. 4): pp. 14-27.

7. Hillner B E, Ingle $J \mathrm{~N}$, Chlebowski $R$ T, et al., "American Society of Clinical Oncology 2003 update on the role of bisphosphonates and bone health issues in women with breast cancer", [published erratum appears in J. Clin. Oncol. (2004), 22: p. 1,351], J. Clin. Oncol. (2003);21: pp. 4,042-4,057.

8. Berenson J R, Hillner B E, Kyle $R A$ et al., "American Society of Clinical Oncology clinical practice guidelines: the role of bisphosphonates in multiple myeloma”, J. Clin. Oncol. (2002);20: pp. 3, 719-3,736.

9. Rosen L S, Gordon D, Kaminski $M$ et al., "Long-term efficacy and safety of zoledronic acid compared with pamidronate disodium in the treatment of skeletal complications in patients with advanced multiple myeloma or breast carcinoma. A randomized, doubleblind, multicenter, comparative trial”, Cancer (2003);98: pp. 1,735-1,744.

10. Kohno N, Aogi K, Minami H et al., "A randomized, double-blind, placebo-controlled phase III trial of zoledronic acid in the prevention of skeletal complications in Japanese women with bone metastases from breast cancer [abstract]”, Proc. Am. Soc. Clin. Oncol. (2004);23: 43, (abstract 668).

11. Body J-J, Diel I J, Lichinitser M R et al., "Intravenous ibandronate reduces the incidence of skeletal complications in patients with breast cancer and bone metastases”, Ann. Oncol. (2003);14: pp. 1,399-1,405.

12. Paterson A H G, Powles TJ, Kanis J A, McCloskey E, Hanson J, Ashley S, "Double-blind controlled trial of oral clodronate in patients with bone metastases from breast cancer", J. Clin. Oncol. (1993);11: pp. 59-65.

13. Kristensen B, Ejlertsen B, Groenvold M, Hein S, Loft H, Mouridsen H T, "Oral clodronate in breast cancer patients with bone metastases: a randomized study”, J. Intern. Med. (1999);246: pp. 67-74.

14. Body J J, Diel I J, Lichinitzer $M$ et al., "Oral ibandronate reduces the risk of skeletal complications in breast cancer patients with 
metastatic bone disease: results from two randomised, placebo-controlled phase III studies”, Br.J. Cancer (2004);90: pp. 1,133-1,137.

15. Rosen L S, Gordon D H, Dugan W Jr et al., "Zoledronic acid is superior to pamidronate for the treatment of bone metastases in breast carcinoma patients with at least one osteolytic lesion”, Cancer (2004);100: pp. 36-43.

16. Rosen L S, Coleman R E, Gordon D et al., "Zoledronic acid is superior to pamidronate in patients with breast cancer and bone metastases [poster]”, Presented at: St. Gallen Oncology Conferences: Primary Therapy of Early Breast Cancer 8th International Conference; March 12-15 2003; St Gallen, Switzerland, poster 100.

17. Williams G, Pazdur R, Temple R, "Assessing tumor-related signs and symptoms to support cancer drug approval”, J. Biopharm. Stat. (2004);14: pp. 5-21.

18. Conte P and Guarneri V, "Safety of intravenous and oral bisphosphonates and compliance with dosing regimens", Oncologist (2004);9 (suppl. 4): pp. 28-37.

19. Lipton A, "Management of metastatic bone disease and hypercalcemia of malignancy", Am. J. Cancer (2003);2: pp. 427-438.

20. Powles T, McCloskey E, Kurkilahti $M$, "Oral clodronate for adjuvant treatment of operable breast cancer: results of a randomized, double-blind, placebo-controlled multicenter trial [abstract]”, Proc. Am. Soc. Clin. Oncol. (2004);23: p. 9 (abstract 528).

21. Saarto T, Vehmanen L, Blomqvist C, Elomaa I, "Ten-year follow-up of a randomized controlled trial of adjuvant clodronate treatment in node-positive breast cancer patients [abstract]”, Proc. Am. Soc. Clin. Oncol. (2004);23: p. 8 (abstract 527).

22. Jaschke A, Bastert G, Solomayer E F, Costa S, Schuetz F, Diel I J, "Adjuvant clodronate treatment improves the overall survival of primary breast cancer patients with micrometastases to bone marrow-a longtime follow-up [abstract]”, Proc. Am. Soc. Clin. Oncol. (2004);23:p. 9 (abstract 529).

23. Hortobagyi G N, Theriault $R$ L, Lipton A, et al., "Long-term prevention of skeletal complications of metastatic breast cancer with pamidronate”, J. Clin. Oncol. (1998);16: pp. 2,038-2,044.

24. Theriault $R$ L, Lipton A, Hortobagyi G N, et al., "Pamidronate reduces skeletal morbidity in women with advanced breast cancer and lytic bone lesions: a randomized, placebo-controlled trial”, Protocol 18 Aredia Breast Cancer Study Group, J. Clin. Oncol. (1999);17: pp. 846-854.

25. Diel IJ, Body J J, Lichinitser M R et al., "Improved quality of life after long-term treatment with the bisphosphonate ibandronate in patients with metastatic bone disease due to breast cancer", Eur. J. Cancer (2004); 40: pp. 1,704-1,712.

26. Body J-J, Diel I, Bell R et al., "Oral ibandronate improves bone pain and preserves quality of life in patients with skeletal metastases due to breast cancer", Pain (2004);111: pp. 306-312.

27. Rizzoli R, "Bisphosphonates and reduction of skeletal events in patients with bone metastatic breast cancer", Ann. Oncol. (2004);15: pp. 700-701.

28. Westermann $A M$, "Intravenous ibandronate reduces the incidence of skeletal complications in patients with breast cancer and bone metastases”, Ann. Oncol. (2004);15: pp. 537-538.

29. Andersen P K, Gill R D, "Cox's regression model for counting processes: a large sample study", Ann. Stat. (1982);10: pp. $1,100-1,120$.

30. Rosen L S, Gordon D, Kaminski M et al., "Zoledronic acid versus pamidronate in the treatment of skeletal metastases in patients with breast cancer or osteolytic lesions of multiple myeloma: a phase III, double-blind, comparative trial”, Cancer J. (2001);7: pp. 377-387.

31. Gnant M, Hausmaninger H, Samonigg H, et al., "Changes in bone mineral density caused by anastrozole or tamoxifen in combination with goserelin ( \pm zoledronate) as adjuvant treatment for hormone receptor-positive premenopausal breast cancer: results of a randomized multicenter trial [abstract]", Presented at: 25th Annual San Antonio Breast Cancer Symposium; December 11-14 2002; San Antonio, Texas (abstract 12).

32. Theriault R, Jakesz R, Gnant M, Gralow J, "The evolving role of bisphosphonates for the prevention of cancer treatment-induced bone loss in patients with breast cancer [abstract]”, Bone (2004);34: pp. S90-S91 (abstract 75).

33. Coleman $R$, Rosen L, Zheng $M$, on behalf of the Study 010 investigators, "Zoledronic acid has long-term efficacy in reducing skeletal complications in patients with bone metastases from breast cancer [abstract]", Presented at: 4th European Breast Cancer Conference, March 16-19 2004; Hamburg, Germany (abstract 246). 